\title{
Discours
}

Revue de linguistique, psycholinguistique et informatique. A journal of linguistics, psycholinguistics and computational linguistics

$6 \mid 2010$

Le fonctionnement en discours des énoncés averbaux autonomes

\section{Équivalents finnois des prédications averbales premières et secondes}

Finnish equivalents of verbless primary and secondary predications

\section{Eva Havu}

\section{OpenEdition}

\section{Journals}

Édition électronique

URL : http://journals.openedition.org/discours/7718

DOI : $10.4000 /$ discours. 7718

ISSN : 1963-1723

Éditeur :

Laboratoire LATTICE, Presses universitaires de Caen

Référence électronique

Eva Havu, «Équivalents finnois des prédications averbales premières et secondes », Discours [En ligne], 6 | 2010, mis en ligne le 29 septembre 2010, consulté le 30 avril 2019. URL : http:// journals.openedition.org/discours/7718; DOI : 10.4000/discours.7718

Ce document a été généré automatiquement le 30 avril 2019.

\section{(c) (i) (9) $\Theta$}

Discours est mis à disposition selon les termes de la licence Creative Commons Attribution - Pas d'Utilisation Commerciale - Pas de Modification 4.0 International. 


\section{Équivalents finnois des prédications averbales premières et secondes}

Finnish equivalents of verbless primary and secondary predications

\section{Eva Havu}

\section{Introduction}

Certains types de prédications averbales premières (prédications autonomes) et secondes semblent à première vue se distinguer les uns des autres seulement par le choix de la ponctuation (ex. [1] ponctuation faible : prédication seconde / ex. [2] ponctuation forte : prédications autonomes) :

[1] Ses bras nus entourent sa tête, abandonnés. Le corps n'est pas convenablement couché comme celui des autres filles, [...]. (Duras, L'Amant)

[1'] Ses bras nus entourent sa tête. Abandonnés. Le corps n'est pas convenablement couché comme celui des autres filles, [...].

[2] Le policier tient entre ses mains une convocation du tribunal pour parents d'enfants délinquants. Il frappera ainsi chez quatre familles. À toutes, il délivrera l'instance du juge. Aimable, presque réconfortant. Mais intraitable. Ces parents d'enfants fugueurs, dealers, voleurs, violents, incapables de contenir leurs rejetons devront suivre un stage dit parental. (Delorme et Lefeuvre, $2004: 291$ )

[2'] [...] À toutes, il délivrera l'instance du juge, aimable, presque réconfortant, mais intraitable. Ces parents d'enfants fugueurs, dealers, voleurs, violents, incapables de contenir leurs rejetons devront suivre un stage dit parental.

2 Lefeuvre (2009) et Delorme et Lefeuvre (2004) ont déjà examiné les différences entre ces deux types de construction et montré que la ponctuation est généralement bien justifiée (cf. changement de sens dans ex. [1'] et [2'] et chapitre 1). Dans cet article, nous étudierons la question surtout d'un point de vue contrastif. Il nous semble en effet fort probable que dans une langue à cas, tel le finnois, le marquage casuel doit également contribuer à souligner l'autonomie ou la « subordination ${ }^{1}$ de la prédication, en plus de 
la ponctuation. Le marquage casuel montrerait-il des différences dans l'autonomie des prédications averbales et, éventuellement, un champ «flou » entre les deux types de prédication?

3 Après une brève présentation des constructions averbales premières et secondes françaises et leurs différences majeures, nous examinerons la manière dont ces prédications se construisent en finnois, pour étudier par la suite les traductions finnoises de constructions averbales apparaissant dans un corpus littéraire.

4 L'emploi de ces prédications est étroitement lié au type de texte: dans la langue des médias elles sont omniprésentes, mais occupent des places et fonctions différentes d'après le type de conception (oral ou écrit). Dans les romans, leur emploi et fonction dépendent beaucoup du type de narration (récit / dialogue) et surtout du style personnel de l'auteur (voir ci-dessous; cf. aussi Havu et Pierrard, 2007, Delorme et Lefeuvre, à paraître). Elles jouent donc un rôle important pour la structuration de différents types de discours dont tous mériteraient d'être l'objet d'une étude contrastive. Le choix d'un corpus littéraire s'explique par l'approche contrastive adoptée ainsi que par les variations personnelles intéressantes apparaissant dans les romans.

\section{Les prédications averbales premières et secondes en français}

5 D'après Lefeuvre (1999) et Delorme et Lefeuvre (2004), sont considérées comme prédications premières averbales des constructions averbales pourvues d'une modalité d'énonciation (assertion, injonction, interrogation) et pouvant donc constituer une phrase. Les prédicats averbaux peuvent être de nature adjectivale [3], nominale [4], prépositionnelle [5] ou adverbiale [6] et être liés à un sujet explicite [3-4] ou implicite [5-6]:

[3] Pas tendre du tout, le sourire. (Pennac ; Lefeuvre, 1999 : 151)

[4] Une merveille, cette machine ! (Lefeuvre, 1999 : 169)

[5] À moi, Brando ! s'écria Orso. (Mérimée ; Lefeuvre, 1999 : 249)

[6] Debout ! (Lefeuvre, 1999 : 169)

Dans les cas où le prédicat averbal pose l'existence d'une situation ou d'un objet, on ne trouve pas de sujet explicite ni implicite :

[7] Hier, confessions. (Bernanos ; Lefeuvre, 1999 : 312)

7 Le référent du sujet peut également être localisé grâce au contexte situationnel [8], ou renvoyer à une prédication antérieure de façon résomptive [9] :

[8] Deux petits verres furent pris dans l'arrière-boutique et apportés sur la planche aux préparations; puis les deux hommes examinèrent en l'élevant vers le gaz la coloration du liquide.

- Joli rubis ! déclara Pierre. (Maupassant ; Lefeuvre, 1999 : 258)

[9] Plus loin, une déléguée FCPE s'emporte haut et fort: «On s'en fiche de savoir qui a raison des Esan ou des PVP. On veut que nos marmots reprennent les cours de natation. La Mairie doit agir!». Pas si facile (Le Nouvel observateur) 

modalité d'énonciation et qui ne s'interprètent qu'en fonction d'un facteur extérieur. Parmi ces constructions incomplètes, elliptiques, on trouve par exemple les réponses aux questions, où le prédicat se construit à l'aide du prédicat de la phrase précédente (Quand viendras-tu? - Demain), ainsi que les ajouts (J'ai acheté deux kilos de cerises. Et des poires) (cf. Lefeuvre, 1999 : 42-43).

9 La prédication seconde, quant à elle, est traditionnellement définie sur un plan sémantico-syntaxique comme «un type de séquence qui, malgré son statut syntaxiquement intégré, exprime sémantiquement un contenu phrastique à l'intérieur même de la phrase » (Cadiot, Furukawa (eds), 2000), avec une relation sous-jacente sujet / prédicat (cf. Combettes, 1998 : Jean est parti, furieux > Jean est parti et il était furieux ). Contrairement à la prédication averbale première, elle n'est pas pourvue d'une modalité d'énonciation (cf. Lefeuvre, 2009), se rattachant obligatoirement à une proposition principale où se trouve, en principe, son point d'ancrage.

10

Dans la description linguistique du français, le terme de prédication seconde est utilisé pour désigner une très large gamme de constructions apparemment assez disparates (voir p. ex. Havu, Pierrard, 2009), mais les seules pouvant éventuellement entrer en "concurrence» avec les prédications autonomes averbales, sont les constructions détachées :

[10a] Jean est parti, furieux.

[10b] Sartre, mon auteur préféré, se vend mal.

11 Comme la prédication première averbale, la prédication seconde peut être de nature adjectivale [11a], nominale [11b], prépositionnelle [11c] ou adverbiale [11d] :
[11a] Jeune, il faisait du sport.
[11b] Ma sœur, une véritable petite demoiselle, ne pense qu'à ses vêtements.
[11c] En grève depuis sept jours, à la suite d'une série d'agressions dont ont été victimes plusieurs des leurs, les convoyeurs de fonds semblent plus que jamais déterminés à tenir jusqu'au bout. (Le Figaro, 15/05/2000 : 12)
[11d] Plus près de chez nous, les jeunes bafouaient l'autorité de l'État incarnée par les forces de l'ordre. (Libération, 16/04/2002 : 15)

La majeure distinction entre les deux types de prédication est donc la présence d'une modalité d'énonciation dans la prédication averbale première et son absence dans la prédication averbale seconde. De plus, la prédication seconde se rattache sans problème au contexte droit, ce qui est exceptionnel pour les prédications premières, et elle est plus facilement objective, la prédication première étant de type plus subjectif (Lefeuvre, 2009 : 358).

Lefeuvre (2009) ainsi que Delorme et Lefeuvre (2004) montrent que la différence de ponctuation influe également sur l'interprétation (voir ex. [13] et [14]). La prédication première forme une sorte de réflexion après coup et constitue souvent un pivot entre le contexte de droite et celui de gauche ${ }^{2}$. La prédication seconde finale complète généralement le contexte de gauche où se trouve également son point d'ancrage, tandis que la prédication seconde initiale a son point d'ancrage dans le contexte de droite, bien que pouvant assurer une continuité informative avec le contexte de gauche (phrase ou contexte précédents) : 
[12] En Afghanistan, la révolte contre les mollahs se développait parmi les femmes. Défiant le pouvoir des talibans, deux jeunes femmes étaient parties faire des études aux USA. (Le Vif, $22^{\mathrm{e}}$ année, $\mathrm{n}^{\circ} 13$ )

\section{une prédication autonome, mais il faut donc y joindre l'assertion, ce qui en change} l'interprétation:

[13] Ses parents se disputent sa garde devant les tribunaux. Ils s'aimaient, avant ; ils se détestent aujourd'hui, et Julie pleure de l'intérieur, coupable et tiraillée. On la voit, perdue dans sa tristesse [...].

[13'] Ses parents se disputent sa garde devant les tribunaux. Ils s'aimaient, avant; ils se détestent aujourd'hui, et Julie pleure de l'intérieur. Coupable et tiraillée. On la voit, perdue dans sa tristesse [...].

15

Dans ce travail où nous nous interrogerons sur la frontière entre les deux types de prédication, seules les prédications secondes détachées en position finale nous intéresseront. En position médiane, elles n'entrent pas en "concurrence » avec une prédication autonome; quant à la position initiale, nous venons de voir que la prédication seconde s'interprète sémantiquement par rapport au contexte droit, tandis que la prédication autonome se rattache généralement au contexte gauche, et de ce fait, elles ne sont pas non plus en « concurrence » :

[14] Olga n'aime à présent plus rien ni personne. Ce qui l'a changée n'est pas tant le cancer que la dépression causée par sa révélation, au nom du dogme sacro-saint de la vérité due au malade. Amère, irascible, capricieuse, jamais satisfaite, depuis un an, elle ne consent plus à se nourrir, n'ouvre plus les yeux, ne s'exprime que par grognements. [...] (Lefeuvre, 2009: 351-352: cause de ne plus consentir à se nourrir, etc.)

[14'] Olga n'aime à présent plus rien ni personne. Ce qui l'a changée n'est pas tant le cancer que la dépression causée par sa révélation, au nom du dogme sacro-saint de la vérité due au malade. Amère, irascible, capricieuse, jamais satisfaite. Depuis un an, elle ne consent plus à se nourrir, n'ouvre plus les yeux, ne s'exprime que par grognements. [...] (Lefeuvre, 2009: 351-352: conséquence de ce qui est signifié dans le contexte gauche)

\section{Les prédications premières et secondes en finnois}

En finnois, les prédications secondes françaises liées et détachées correspondent à des 'compléments prédicatifs', qui sont marqués par des cas spécifiques, tels que l'essif, mais pas par la ponctuation :

[15a] Il boit son café chaud.

Hän juo kahvinsa kuumana (essif).

[15b] Enfant, Jean était toujours malade.

Lapsena (essif) Jean oli aina sairas.

[15c] Il boit son café chaud.

Hän juo kuuman kahvinsa (les deux éléments du SN sont à l'accusatif).

Les prédications secondes détachées purement descriptives, courantes en français, ne peuvent cependant pas être traduites par un essif; on doit avoir recours à une 
subordonnée relative ou bien à une construction épithétique, qui, toutefois, fait disparaître le contenu prédicatif :

[15d] Âgé d'un peu plus de 35 ans, Kamel ben Sallah est soupçonné d'avoir participé à cette boucherie commise au fusil de chasse et au couteau. (Le Figaro, 15/05/2000: 12)

Kamel ben Sallahia, joka onhieman yli 35-vuotias, epäillään [...] [= qui est]. Hieman yli 35-vuotiasta Kamel ben Sallahia epäillään [...] [= 'L'un peu plus de 35 ans vieux' KbS est soupçonné de]. vient de se marier), et la différence entre les constructions détachées et liées françaises (ex. [15a] et [15b]: place) ainsi que celle entre une prédication seconde et une épithète (ex. [15a] et [15c]: place et cas) se manifeste donc généralement par d'autres moyens syntaxiques et / ou casuels.

On trouve également en finnois des prédications averbales autonomes (« finiittiverbittömät lausemaiset rakenteet » 'constructions phrastiques sans verbe fini' ; Hakulinen et al., 2004: §879; cf. Helasvuo, 1997, qui, toutefois ne traite que des constructions nominales, «Free NPs » $\left.{ }^{3}\right)$. Même si le finnois a un système casuel très riche (14-16 $\left.\mathrm{cas}^{4}\right)$, les constructions prédicatives nominales et adjectivales sont normalement au nominatif ou au partitif (cf. Hakulinen et al., 2004), le premier étant un cas sujet par excellence, et le deuxième étant généralement un cas objet, mais pouvant marquer également un sujet ${ }^{5}$. L'emploi majoritaire de ces deux cas est confirmé par un corpus de romans français traduits en finnois et par un corpus de romans d'auteurs finlandais (Havu, 2009). Helasvuo $(1997$ : 137, 141) mentionne les mêmes cas pour les SN, et constate que certains cas, tels que le génitif / accusatif, n'y apparaissent presque jamais, ce qui s'explique probablement par leur dépendance à un autre élément (p. ex. les accusatifs marquent des arguments verbaux). En plus des constructions averbales SN et SA, on trouve en finnois des constructions averbales Spost- ou prépositionnels ou des syntagmes à verbe non fini (infinitifs, participes), qui ne seront pas traités dans cet article :

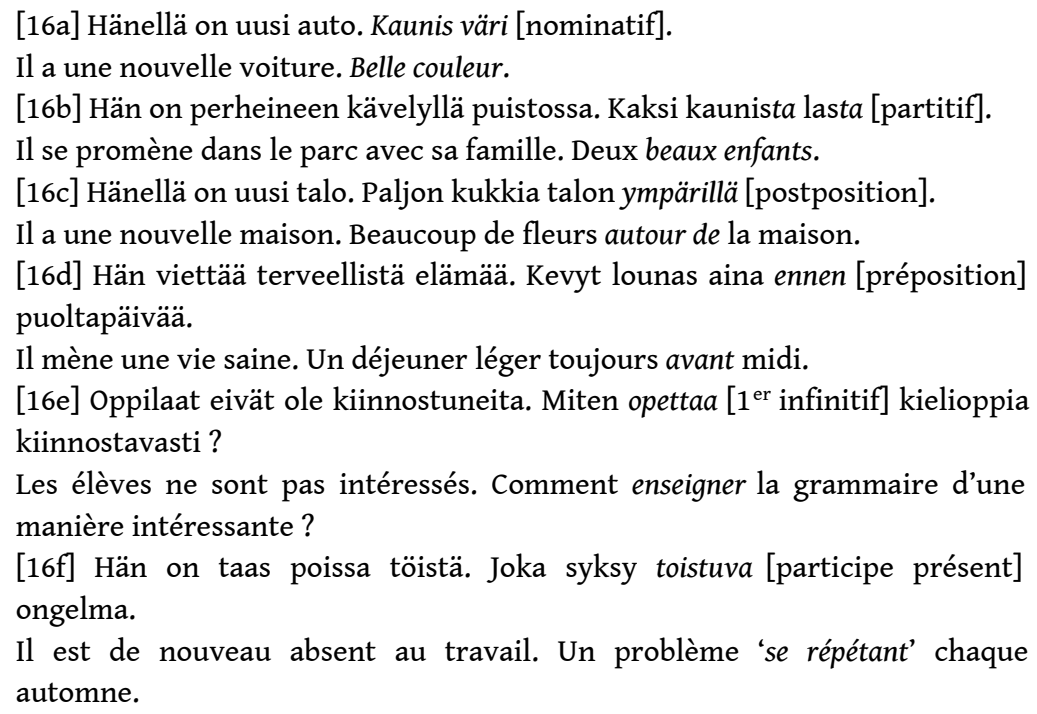

Hakulinen et al. (2004 : § 879, 881, 1021) mentionnent différents contextes d'emploi de ces constructions, tels que les consignes et directives (Toinen ovi vasemmalla 'Deuxième porte à gauche', Yksi olut 'Une bière'), titres ${ }^{6}$ (Ahtisaari uudestaan valtiovierailulle Moskovaan 
'Ahtisaari de nouveau en visite officielle à Moscou' > 'Ahtisaari [part] de nouveau en visite officielle à Moscou'), commentaires (Todella hyvää tämä keitto 'Vraiment bonne, cette soupe') ou exclamations (Kuinka ihana aamu 'Quel beau matin'). Les réponses aux questions ne sont pas considérées comme des prédications premières indépendantes (Helasvuo, 1997 : 141), ce qui était également le cas en français.

21 Nous examinerons ci-dessous les équivalents finnois de prédications averbales premières et secondes adjectivales (adjectifs et participes passés) et nominales apparaissant dans trois romans français ${ }^{7}$, pour voir comment les traducteurs ont traité la différence de ponctuation au niveau casuel : les prédications secondes, « subordonnées » à un élément de droite ou de gauche, sont-elles à un cas autre que le nominatif (et le partitif), et les prédications premières, par principe autonomes, sont-elles surtout au nominatif (et au partitif)?

\section{3. Équivalents finnois de prédications premières averbales}

Des trois textes examinés, celui de Gavalda contient le plus de prédications averbales, tandis que celui d'Echenoz en contient le moins. Dans deux ouvrages, la quantité de prédications autonomes est en distribution complémentaire avec celle des prédications secondes : les prédications secondes sont de loin les plus courantes chez Echenoz, tandis que chez Gavalda, elles sont peu nombreuses. Chez Duras, en revanche, les deux sont bien représentées.

Gavalda a un style "saccadé ", avec de nombreux paragraphes très courts, et les prédications autonomes commencent ou clôturent généralement ces paragraphes :

[17] [Début du paragraphe] Ces maudits engins [portable], il en faut toujours un, n'importe où, n'importe quand. Un goujat. [fin du paragraphe] (Gavalda : 19)

Jollakulla on sitten aina tuollainen kirottu kapistus, oltiinpa missä tahansa, milloin tahansa. Moukka. (Schuurman : 16)

La tendance à clôturer par une prédication averbale se voit également chez les autres auteurs, mais d'une manière moins importante (cf. ex. [20b]).

Les trois traductions montrent des différences : le traducteur d'Echenoz a transformé la plupart des constructions averbales en constructions verbales (ajout d'un verbe: ex. [18a]), tandis que les traducteurs de Duras et de Gavalda ont essayé de respecter les constructions françaises, et les changements de la structure de la phrase de départ sont très rares (cf. ex. [18b]) :

[18a] Puis, le portail franchi, négligeant l'ascenseur, il attaqua fermement un escalier de service. Il parvint au sixième étage moins essoufflé que j'aurais cru, devant une porte mal repeinte en rouge brique et dont les montants témoignaient d'au moins deux tentatives d'effraction. Pas de nom sur cette porte, juste une photo punaisée, gondolée aux angles et représentant le corps sans vie de Manuel Montoliu, ex-matador recyclé péon, [...] : Ferrer frappa deux coups légers sur cette photo. (Echenoz : 9)

Päästyään sisään hän ohitti hissin ja hyökkäsi lujasti rappusiin. Kun hän saapui seitsemänteen kerrokseen, hän ei ollut niin hengästynyt kuin olisi luullut ja pysähtyi kehnosti maalatulle tiilenpunaiselle ovelle, jonka 
karmeihin oli jäänyt ainakin kahden murtoyrityksen jäljet. Ovessa ei ollut nimeä [Sur la porte, il n'y avait pas de nom] vain nastalla kiinnitetty ja nurkistaan käpristynyt valokuva Manuel Montoliu-nimisen maatyöläisen, uusiokäytössään matadorin elottomasta ruumiista, [...]; Ferrer koputti valokuvaa kevyesti kaksi kertaa. (Jukarainen : 6)

[18b] Je ne danse jamais avec mon frère aîné, je n'ai jamais dansé avec lui. Toujours empêchée par l'appréhension troublante d'un danger, celui du rapprochement de nos corps. (Duras : 68)Mutta isoveljeni kanssa minä en tanssi koskaan, en ikinä tanssinut hänen kanssaan. Esteenä oli aina [Comme empêchement il y avait toujours] hämmentävä vaaran pelko, hänen turmiollisen vetovoimansa, ruumiidemme kosketuksen pelko. (Mannerkorpi : 63) nominatif [20a-c] ou au partitif [21a-b], cas qui apparaît donc obligatoirement après une négation ou un numéral et qui est utilisé pour exprimer une quantité non dénombrable :

[20a] Les repas étaient commandés chez les meilleurs traiteurs de Paris. Toujours décents, presque, mais à peine, insuffisants. (Duras : 80)Ateriat tilattiin Pariisin parhailta ravintoloitsijoilta. Aina moitteettomat ruoat [nominatif], ainakin melkein, mutta niukat, riittämättömät [nominatifs]. (Mannerkorpi : 71) [20b] Puis des masques en tous genres et, pour finir, une collection de crânes aux bouches colmatées par des rails d'obsidienne, aux orbites obturées par des boules d'ivoire de morse incrustées de pupilles en jais. Une fortune. [fin de paragraphe] (Echenoz : 84-85) :

Sitten on kaikenlaisia naamioita ja vihdoin kokoelma kalloja, joiden suut oli suljettu obsidiaanitapeilla ja silmäkuopat tukittu gagaattikoristeisilla mursunluukuulilla. Kokonainen omaisuus[nominatif]. (Jukarainen :72)

[20c] Je retrouve tous mes esprits. D'un coup.

Le traitre.

L'ingrat.

Qu'as-tu donc fait là malheureux !!! (Gavalda : 21)

Olen taas täysissä tolkuissani. Yks kaks.

Petturi [nominatif].

Kiittämätön [nominatif].

Mitä sinä onneton menit tekemään !!! (Schuurman : 18)

[21a] L'air était bleu, on le prenait dans la main. Bleu. (Duras : 100)

Ilma oli sinistä, sitä voi käsin kosketella. Sinistä [partitif]. (Mannerkorpi : 87)

[21b] À dix-neuf heures j'ai regardé les infos régionales. L'horreur. Huit morts et soixante blessés. [...] (Gavalda : 103-104)

Seitsemältä illalla katsoin televisiosta paikallisuutiset. Karmeaa. Kahdeksan kuollutta ja kuusikymmentä loukkaantunutta [partitifs].[...] (Schuurman : 90)

28

Très rarement, et presque uniquement dans la traduction d'Echenoz, on trouve un autre cas, ce qui montre que le traducteur a vu dans la prédication séparée par la ponctuation forte une subordination par rapport à un élément de la phrase précédente. Dans [22a], le cas inessif, qui correspond ici à la préposition «sur ", apparaît déjà dans le contexte de 
gauche et souligne le fait qu'il s'agit dans le texte de départ d'un ajout, et donc pas vraiment d'une prédication autonome :

[22a] Délaissant insensiblement les plasticiens, il continuait bien sûr à s'occuper de ses peintres, surtout Gourdel et Martinov - [...] - mais il envisageait maintenant de porter le gros de ses efforts sur des pratiques plus traditionnelles. Art Bambara, art bambou, art indien des plaines et toute cette sorte de choses. Pour le conseiller dans ses investissements, il s'était assuré les services d'un informateur compétent [...]. (Echenoz : 26)

Hylättyään tylysti plastiset taiteilijat hän huolehti edelleen maalareistaan, [... ], mutta aikoi keskittää ponnistuksensa vastedes perinnevoittoisempiin taidemuotoihin [inessif]. Bambarataiteeseen, bantutaiteeseen, tasankointiaanien taiteeseen ja muihin vastaaviin [inessifs]. Saadakseen neuvoja sijoitusasioissa hän oli värvännyt avustajakseen erään Delahaye-nimisen asiantuntijan [...]. (Jukarainen : 22)

Dans la traduction finnoise de [22b] apparait également un inessif qui correspond aux prépositions 'à' / 'dans'. Dans la version française, une préposition ne pourrait guère être ajoutée à la construction autonome, tandis qu'en finnois, la prédication autonome reprend l'inessif de la phrase précédente et se transforme en une structure "subordonnée ": Je trouve ridicule que tu exposes à la Caisse des dépôts et consignations. Et encore [que tu exposes] $\grave{a}>$ dans une exposition de groupe :

[22b] Personnellement, dit Ferrer, je trouve ridicule que tu exposes à la Caisse des dépôts et consignations. Ridicule. Une exposition de groupe en plus. Tu te dévalues. (Echenoz : 45)

Henkilökohtaisesti, sanoi Ferrer, minusta on naurettavaa, että sinä annat töitäsi näytteille vakuus- ja panttikassaan [inessif]. Naurettavaa. Ja vielä ryhmänäyttelyyn [inessif]. Myyt itsesi liian halvalla. (Jukarainen : 38)

Les traducteurs, surtout ceux de Duras et de Gavalda, suivent donc étroitement le modèle de la langue source, et les prédications averbales détachées par un point ne sont que très rarement transformées en des prédications subordonnées à un élément de la phrase précédente.

\section{4. Équivalents finnois de prédications secondes détachées}

Nous n'avons pu inclure dans le corpus des prédications secondes finales que des SA (adjectifs ou participes passés), car les SN n'apparaissent guère en position finale (Mon frère, un enseignant dévoué, prendra un mois de congé > ??? Mon frère prendra un mois de congé, un enseignant dévoué; Enseignant dévoué, mon frère est toujours joignable > ?? Mon frère est toujours joignable, enseignant dévoué). Comme nous l'avons déjà constaté, ces prédications sont proportionnellement les plus courantes chez Echenoz où le taux des prédications indépendantes est le plus bas, et elles sont les moins courantes chez Gavalda, où les prédications indépendantes sont les plus nombreuses. Traduits aussi littéralement que possible, les SA finnois correspondant aux prédications secondes ayant une valeur circonstancielle en français (temporelle, de cause, de concession...) devraient être à l'essif, et les prédications secondes purement descriptives devraient être traduites par une subordonnée relative (voir ci-dessus). 

texte source que celles des prédications premières, et rares sont les cas où un SA finnois a été choisi dans la traduction d'un SA français. Souvent, la prédication averbale participiale (très rarement, la construction adjectivale) a été transformée en une construction verbale (verbe ajouté : [23a]) ou bien la phrase a été entièrement reformulée [23b-c] :

[23a] Dans l'ombre de ces villas, elles se regardent pour plus tard, elles croient vivre un roman, elles ont déjà les longues penderies pleines de robes à ne savoir qu'en faire, collectionnées comme le temps, la longue suite des jours d'attente. (Duras : 27)

Huviloidensa hämärässä he katsovat itseään myöhemmän varalta, he luulevat elävänsä romaania, heillä on leveät vaatekaapit pukuja pullollaan niin etteivät he tiedä mitä niillä tekisivät, niitä vainkeräillään [elles sont seulement collectionnées] kuin aikaa, odotuspäivien pitkää helminauhaa. (Mannerkorpi : 28)

[23b] Le corps du navire et le gréement semblaient en bon état, pelliculés de gel et d'apparence cassante comme du bois mort. (Echenoz : 81)

Runko ja takila näyttivät olevan hyvässä kunnossa, mutta vaikuttivat kuurakuorrutuksen takia haurailta kuin laho puu[mais à cause de la pellicule de gel, ils semblaient fragiles comme du boismort]. (Jukarainen : 69)

[23c] En voulant traverser l'autoroute pour porter secours aux blessés, deux autres personnes sont tuées, fauchées par une voiture. (Gavalda : 106)

Lisäksi kaksi ihmistä jäi auton alle ja sai surmansa yrittäessään päästä moottoritien yli auttamaan loukkaantuneita [De plus, deux personnes ont été renversées par une voiture et ont été tuées en essayant de traverser l'autoroute pour porter secours aux blessés]. (Schuurman : 93)

La transformation d'une prédication seconde avec valeur circonstancielle en une proposition est courante, surtout dans le cas des constructions à interprétation causale, même si l'essif finnois a lui-même aussi plusieurs valeurs circonstancielles, dont la valeur causale :

[24a] Mais ce soir-là Ferrer, à vrai dire, n'avait guère accordé d'attention à ce récit, trop intéressé par cette Victoire dont il n'imaginait pas qu'elle viendrait s'installer chez lui dans une semaine. (Echenoz : 31 )

Mutta tuona iltana Ferrer ei suonut kertomukselle totta puhuen paljonkaan huomiota, sillä hän oli liian kiinnostunut [car il était trop intéressé par Victoire] Victoiresta eikä osannut kuvitella, ettätämä tulisi asumaan hänen luokseen viikon sisällä. (Jukarainen : 26)

Comme nous l'avons mentionné ci-dessus, la traduction par une subordonnée relative est la seule solution dans le cas des prédications secondes purement descriptives, car l'essif n'a pas cette valeur; une autre solution est de transformer le SA prédicatif en un SA épithétique en choisissant le cas grammatical du SN qu'il décrit, mais dans ce cas, la valeur prédicative disparaît entièrement :

[24b] Deux ou trois fois on aperçut des villages désertés sur les rivages du Labrador, construits à l'origine par le gouvernement central pour le bienfait des autochtones et, de la centrale électrique à l'église, parfaitement équipés. (Echenoz: 22)

Pariin kolmeen otteeseen Labradorin rannalla näkyi hylättyjä kylïa, jotka keskushallitus oli rakentanut [que le gouvernement central avait construits] alkuperäisväestön siunaukseksi javarustanut ne täydellisesti kirkosta 
sähkövoimalaan saakka. (Jukarainen: 19)> Pariin kolmeen otteeseen Labradorin rannalla näkyi keskushallituksen alkuperäisväestön siunaukseksi rakentamia hylättyjä kyliä [des villages désertés construits par le gouvernement central pour le bienfait des autochtones]. épithétique est courante si la prédication seconde est un adjectif. Dans l'exemple [25c], nous trouvons le même adjectif dans une prédication seconde et dans une prédication autonome. Dans le premier cas, il a été transformé en une épithète, tandis que dans le deuxième, il est autonome et au partitif. La construction sous-entendue en finnois serait ici "c'est incontrôlable/incroyable»; si on sous-entendait que la femme est incontrôlable, la prédication autonome devrait être au nominatif (hillitön) :

[25a] Jalouse elle est. Pas de réponse, un regard bref aussitôt détourné, le petit haussement d'épaules, inoubliable. (Duras : 31 )

Kateellinen hän vain oli. Ei vastausta, hätäisesti pois käännetty katse, unohtumaton pieni olankohautus [un inoubliable petit haussement d'épaules].

(Mannerkorpi : 31)

[25b] Puis le cours des choses reprit, interminable. (Echenoz : 35)

Sen jälkeen tapahtumat jatkoivat samaa loputonta kulkuaan [Puis les événements reprirent leur interminable cours]. (Jukarainen : 29)

[25c] La voilà partie dans un show d'enfer, incontrôlable. Elle se dandine, mime un strip-tease, renifle la culotte, se retient à l'halogène et tombe à la renverse.

Incontrôlable. (Gavalda : 181)

Ja hän heittäytyi aivan hillittömään showhun [dans un incontrôlable / incroyable show]. Hän keikutteli lanteitaan, oli strippaavinaan, nuuhkaisi pikkuhousuja, otti tukea halogeenilampusta ja kaatui taaksepäin.

Hillitöntä [Incontrôlable / incroyable]. (Schuurman : 159) trouve effectivement assez souvent dans les traductions de Duras, mais très sporadiquement dans les deux autres traductions. Les participes passés sont ici bien plus courants que les adjectifs [26a-b]. Généralement, les constructions à l'essif ne sont jamais détachées en finnois, mais la traductrice de Duras [26d] et le traducteur d'Echenoz [26e] ont eu recours au détachement une fois chacun dans une construction participiale très longue. La traduction de Duras est exceptionnelle aussi dans la mesure où il s'agit d'un essif causal, les autres essifs utilisés dans la traduction exprimant normalement la manière :

[26a] Nous restons ainsi, cloués, à gémir dans la clameur de la ville encore extérieure. (Duras : 58)

Me jäämme makaamaan toisiimme naulittuina, voihkimaan keskellä ulkopuolelta yhä kantautuvaa kapunkimelua. (Mannerkorpi : 53)

[26b] Je le regarde, amusée. (Gavalda : 13)

Katselen häntä huvittuneena. (Schuurman : 10)

[26c] Sa grande automobile était là, longue et noire, avec, à l'avant, le chauffeur en blanc. (Duras : 135)

Miehen suuri auto seisoi satamassa pitkänä ja mustana, etupenkillään valkopukuinen kuljettaja. (Mannerkorpi : 116)

[26d] À la libération de Paris, poursuivi sans doute pour faits de collaboration dans le Midi, il ne sait plus où aller. (Duras : 95)

Pariisin vapautuksen aikaan, takaa-ajettuna ilmeisesti yhteistoiminnasta 
saksalaisten kanssa Etelä Ranskassa, hän ei tiedä minne mennä. (Mannerkorpi : 83)

[26e] Tout semblait bien là comme prévu, serré dans trois grosses cantines métalliques qui avaient honnêtement résisté au temps. (Echenoz : 81)

Kaikki näytti olevan tallessa niin kuin pitikin, ahdettuna kolmeen suureen metalliseen matka-arkkuun, jotka olivat kestäneet säät kunnialla. (Jukarainen : 70) adjectifs ont été directement transformés en un adverbe, tandis que les participes ont été remplacés par une autre expression adverbiale [27c], sans doute plus naturelle dans la traduction d'une forme gardant des traces de son origine verbale ${ }^{9}$ :

[27a] Et elle, lente, patiente, elle le ramène vers elle et elle commence à le déshabiller. (Duras : 49)

Hitaasti ja kärsivällisesti tyttö ohjaa hänet takaisin lähelleen ja alkaa riisua häntä [lentement et patiemment]. (Mannerkorpi : 45)

[27b] Il lui a répondu, faussement distrait, en la faisant tourner sur ellemême. (Gavalda : 123)

Nuorukainen vastasi muka hajamielisesti samalla kun pyöritti tyttöä : [...] [faussement distraitement]. (Schuurman : 107)

[27c] Elle est là où il faut qu'elle soit, déplacée là. (Duras : 47)

Tyttö on juuri siellä missä hänen nyt pitääkin olla, oikealla tavalla poissa paikaltaan[ici : « hors de sa place », au lieu de déplacer = 'siirtää’ > 'siirrettynä']. (Mannerkorpi : 44)

\section{Conclusion}

Dans cet article, nous nous sommes demandé si le système casuel finnois marquait plus clairement que la seule ponctuation les différences entre les prédications autonomes et les prédications secondes: les prédications autonomes seraient-elles à des cas qui ne montrent (généralement) pas de dépendance argumentale par rapport à la phrase précédente (nominatif, partitif) et les prédications secondes à des cas qui en montrent une (génitif / accusatif, essif) ? Pour répondre à cette question, nous avons examiné la traduction finnoise des prédications autonomes et secondes adjectivales et nominales dans trois romans français contemporains.

Nous avons pu constater que les traducteurs, surtout ceux de Duras et de Gavalda, suivent étroitement le modèle de la langue source : le plus souvent, la prédication averbale est, en finnois, également détachée par un point et elle est au nominatif ou au partitif. Le traducteur d'Echenoz modifie plus souvent les constructions averbales de base en y ajoutant un verbe.

Dans la traduction des prédications secondes, les traducteurs ont pris beaucoup plus de libertés, et il est assez rare de trouver une traduction directe, c'est-à-dire une construction adjectivale à l'essif, dans les traductions d'Echenoz et de Gavalda, tandis qu'elles apparaissent plus souvent dans la traduction de Duras. Les participes passés, qui gardent une trace de leur origine verbale, se comportent d'une manière différente des adjectifs :

1. la prédication participiale a souvent été transformée en une construction verbale, ou bien la phrase a été entièrement reformulée; les constructions à l'essif, qui correspondraient le plus aux formes de départ, sont plus courantes que dans le cas des adjectifs; si le traducteur 
a vu dans la construction une valeur adverbiale, il a choisi un adverbe sans lien avec le participe (d'ailleurs, cette interprétation adverbiale n'apparaît jamais dans les constructions autonomes) ;

2. l'adjectif prédicatif a souvent été transformé en un adjectif épithète; les constructions à l'essif sont rares, mais il n'est pas exceptionnel que les traducteurs aient transformé l'adjectif de départ en un adverbe (Il dort, tranquille > Il dort tranquillement).

41 Si l'on ne prend pas en considération les transformations de la phrase entière, les cas / formes les plus fréquents employés dans la traduction des prédications autonomes et des prédications secondes (« subordonnées ») sont les suivants :

1. prédication averbale : nominatif, partitif ;

2. prédication seconde : essif, adverbe, construction épithétique ou relative descriptive.

Même si les traducteurs n'ont pas toujours traduit directement les constructions averbales autonomes et "subordonnées » (prédications secondes), on voit qu'ils ont généralement pris en considération la nature différente de ces deux types de construction, obéissant en cela clairement au marquage de ponctuation des phrases de départ : les prédications autonomes sont à des cas qui ne montrent pas (obligatoirement) une dépendance par rapport au contexte antérieur, tandis que les prédications secondes sont généralement «subordonnées » à un élément dans la phrase même. De plus, les interprétations adverbiales où les traducteurs ont attribué à la prédication seconde un ancrage verbal montrent la frontière parfois floue entre les adjectifs et les adverbes.

\section{BIBLIOGRAPHIE}

CADIOT, P., FURUKAWA, N. (eds) 2000. La prédication seconde. Langue française 127.

COMBETTES, B. 1998. Les constructions détachées en français. Paris : Ophrys.

CRISTOFARO, S. 2003. Subordination. Oxford Studies in Typology and Linguistic Theory. Oxford:

Oxford University Press.

DELORME, B., LEFEUVRE, F. 2004. De la prédication seconde à la prédication autonome. Verbum XXVI : 281-297.

DELORME, B., LEFEUVRE, F. à paraître.Are verbless sentences sentences without verbs ?

HAKULINEN, A.et al. 2004. Iso suomen kielioppi. Helsinki : Suomalaisen kirjallisuuden seura.

HANSEN, G., CHESTERMAN, A., GERZYMISCH-ARBOGAST, H. (eds) 2009. Efforts and Models in Interpreting and Translation Research : A tribute to Daniel Gile. Amsterdam / Philadelphia : John Benjamins.

HAVU, E. 2009. Prédications averbales en finnois : subordination ou autonomie ? Cahiers d'études hongroises 15 : 207-216.

HAVU, E., PIERRARD, M. 2007. Prédication seconde et type de discours : les adjoints participiaux dans les médias oraux. In M. BROTH, M. FORSGREN, C. NORÉN, F. SULLET-NYLANDER (eds), Le français parlé des médias, Actes du colloque de Stockholm, 8-12 juin 2005. Stockholm : Almqvist \& Wiksell International : 273-288. 
HAVU, E., PIERRARD, M. 2009. La prédication seconde : essai de mise au point. Travaux de linguistique $57: 7-21$.

HELASVUO, M.-L. 1997. When discourse becomes syntax : Noun phrases and clauses as emergent syntactic units in Finnish conversational discourse. Dissertation for the degree of Doctor of Philosophy in Linguistics. University of California, Santa Barbara [à consulter p. ex. à la bibliothèque de l'Université de Helsinki].

LEFEUVRE, F. 1999. La Phrase averbale en français. Paris : L'Harmattan.

LEFEUVRE, F. 2009. Segments averbaux isolés : prédication seconde ou première ? In D. APOTHÉLOZ, F. NEVEU (eds), Les linguistiques du détachement. Berne : Peter Lang : 347-359.

VAN RAEMDONCK, D. 2005. L'adverbe, adjectif du verbe ? Ré-partition des rôles. In J. GOES (ed.), L'Adverbe : un pervers polymorphe. Arras : Artois Presses Université.

\section{NOTES}

1. Nous partons du principe que les prédications secondes sont subordonnées à la proposition principale dans la mesure où elles sont dans un rapport de dépendance envers une prédication supérieure, cf. Cristofaro, 2003.

2. La dernière fois, une délégation de salariés est venue la voir. Inquiets. Le patron de l'usine était en train de déménager les machines en douce (dans Delorme et Lefeuvre, 2004 : 284, 287).

3. Les "free NPs» ne forment pas seulement des prédications averbales, mais également des unités référentielles, correspondant à des segments disloqués et appelés « Topic constructions » (Helasvuo, 1997: 153).

4. Le nombre des cas varie d'après les critères employés.

5. P. ex. un numéral ou une négation sont presque toujours accompagnés d'un partitif: Musta kahvi [nominatif] on hyvää 'Le café noir est bon' / Kaksi mustaa kahvia [partitif] on tarjottimella 'Deux cafés noirs sont sur le plateau'; dans certains cas, tels que les ordres / directives, l'objet est au nominatif: Osta auto/Sinun täytyy ostaa auto 'Achète une voiture / Il te faut acheter une voiture'.

6. Helasvuo (1997) ne considère pas les titres comme des prédications averbales : « because they do not exhibit the kind of grammatical structuring that is characteristic of clauses ».

7. Marguerite Duras (1984), L'Amant. Les Éditions de Minuit, Paris ; Rakastaja, traduction par Jukka Mannerkorpi, Otava, Helsinki, 1987.

Jean Echenoz (1999), Je m'en vais. Les Éditions de Minuit, Paris (100 premières pages) ; Minä lähden, traduction par Erkki Jukarainen, Kustannusosakeyhtiö Tammi, Helsinki, 2001.

Anna Gavalda (1999), Je voudrais que quelqu'un m'attende quelque part. Le Dilettante, Paris ; Kunpa joku odottaisi minua jossakin, traduction par Titia Schuurman, Gummerus, Jyväskylä - Helsinki, 2001.

8. On pourrait éventuellement les considérer comme des stratégies de compensation, cf. p. ex. Hansen, Chesterman et Gerzymisch-Arbogast (eds), 2009.

9. P. ex. paraphrase par un auxiliaire et non par une copule : Malheureux, il part en voyage : Il est malheureux / Arrêté pour cambriolage, il ne peut pas partir en voyage : Il est / a été arrêté ; d'ailleurs, on trouve parfois environ le même champ d'emploi entre participes présents prédicatifs, "formes adjectivales ", ayant un ancrage nominal, et gérondifs, «formes adverbiales », ayant un ancrage verbal : Rentrant chez lui, Jean a vu Paul (Jean est rentré et il a vu Paul) / En rentrant chez lui, Jean a vu Paul (simultanéité de 'voir' et de 'rentrer') (cf. Havu et Pierrard, 2009) ; cf. aussi Van Raemdonck, 2005 : «L'adverbe, adjectif du verbe?». 


\section{RÉSUMÉS}

En finnois, les prédications secondes françaises liées et détachées correspondent à des " compléments prédicatifs ", qui sont marqués par des cas spécifiques, tels que l'essif (Hakulinen et al., 2004). Comme le finnois ne connaît que le détachement appositif nominal (Jean, mon frère aîné, vient de se marier), la différence entre les constructions détachées et liées françaises se manifeste par d'autres moyens syntaxiques.

On trouve en finnois également des prédications averbales autonomes qui sont généralement aux cas nominatif et partitif. Toutefois, comme d'autres cas peuvent également apparaître, nous nous demandons si dans la traduction finnoise de romans français les différences entre les prédications averbales clairement autonomes et les ajouts et ellipses sont soulignées par le système casuel et si les prédications autonomes se distinguent des prédications secondes par le choix des cas: les prédications autonomes seraient-elles à des cas qui ne montrent pas obligatoirement une dépendance par rapport à la phrase précédente et les prédications secondes à des cas qui en montrent une ? Le corpus examiné se constitue de la traduction finnoise des prédications autonomes et secondes adjectivales et nominales apparaissant dans trois romans français contemporains.

In Finnish, French detached and integrated secondary predications correspond to so-called "predicative complements", which are marked by a special case, such as the essive (Hakulinen \& alii, 2004). Since only nominal appositions are detached in Finnish (John, my elder brother, has just got married), the difference between French detached and integrated constructions is marked by other syntactic means.

Verbless autonomous predications also appear in Finnish and they normally take the nominative or partitive case. However, since other cases may also appear, we try to find out if in the Finnish translations of French novels the choice of the grammatical case underlines the differences between verbless predications and additions or elliptic adjuncts and between autonomous and secondary predications: do cases used in autonomous predications show more autonomy in relation to the preceding sentence than those appearing in secondary predications? The corpus consists of the Finnish translation of adjectival and nominal autonomous and secondary predications taken from three contemporary French novels.

\section{INDEX}

Mots-clés : prédication autonome averbale, prédication seconde, système casuel du finnois, traduction du français en finnois

Keywords : autonomous verbless predications, Finnish case marking, secondary predications, translation from French into Finnish 


\section{AUTEUR}

\section{EVA HAVU}

Université de Helsinki / Sorbonne Nouvelle 